\title{
PSEUDO-NORMAL FORMS AND THEIR APPLICATIONS
}

\author{
AMADEU DELSHAMS AND J. TOMÁS LÁZARO \\ Departament de Matemàtica Aplicada I, \\ Universitat Politècnica de Catalunya, Barcelona (Spain) \\ E-mail: amadeu@ma1.upc.es, lazaro@ma1.upc.es
}

\section{Introduction and Main Results}

Since they were introduced by Poincaré in his thesis, Normal Forms have become a common and useful tool in the local qualitative study of dynamical systems. Consequently, the literature about this subject is very rich, not only because of the people working on it (Poincaré, Dulac, Birkhoff, Stenberg, Chen, Arnold, Moser, Tokarev, Bibikov, Belitskiǐ, Bruno, Walcher, Cicogna, Gaeta, Bambusi and many others) but the also the amount of publications (see, for instance ${ }^{1,2,6,3,7}$ and references therein).

Let us consider a real planar analytic system

$$
\dot{z}=F(z)=\Lambda z+\hat{F}(z),
$$

with the origin being an equilibrium point and where^means order greater or equal than 2. It is very well known that a transformation leading system (1) into Poincaré-Dulac normal form (or Birkhoff-Gustavson normal form in the Hamiltonian case), consisting only in resonant terms, does not need to converge in a neighborhood of the origin. In particular, this the case if we assume $\Lambda=D F(0)$ to have purely imaginary eigenvalues, which must be conjugated, $\pm \alpha i, \alpha>0$. In such situation, the normal form becomes of type

$$
\left\{\begin{array}{l}
\dot{\xi}=\xi A(\xi \eta) \\
\dot{\eta}=-\eta A(\xi \eta)
\end{array}\right.
$$

with $A(I)=i \alpha+\ldots$ or, in real coordinates, defining $r=\xi \eta=\xi \bar{\xi}$,

$$
\dot{r}=r G(r) \text {. }
$$

Nevertheless there always exists an infinitely differentiable change of coordinates normalizing this kind of systems (Tokarev ${ }^{18}$, Belitskii ${ }^{4}$ ), it is also known that such transformation $z=\Phi(\zeta)$ diverges if the origin is a focus (for a detailed discussion about planar normal forms and convergence of the normalizing transformations, see ${ }^{6}$ ). Completely different is the case in which the eigenvalues of $\Lambda$ are real where we have convergence. For instance, for an analytic Hamiltonian system of the plane, this was proved by Moser ${ }^{14}$. 
In this work, our aim is to approach the problem of the convergence of a normalizing transformation in the case of purely imaginary conjugated eigenvalues. To do it we will deal with an extension of the classical normal form, which will be convergent even in the case of the origin being a focus. Concretely, this extension will be represented by a remainder term, depending only on an scalar (analytic) function containing the obstructions for the integrability of the system. This approach, that comes from ideas of Moser and DeLatte ${ }^{8}$, consists in the following: let us first write (Birkhoff) normal form system (2) in the shorter way $\dot{\zeta}=N(\zeta)$, where $N(\zeta)=(\xi A(\xi \eta),-\eta A(\xi \eta))$; having a (close to identity) change of variables $z=\Phi(\zeta)$ normalizing system (1) implies that we can solve (at least formally) equation $N=\Phi^{*} F=(D \Phi)^{-1}(F \circ \Phi)$ or, equivalently, $D \Phi N=F \circ \Phi$. Instead of dealing with it, we will look for analytic vector fields $N$ and $\hat{B}=(\xi b(\xi \eta), \eta b(\xi \eta)$, and change of variables $\Phi$ in such a way that they satisfy the following equality

$$
D \Phi N+\hat{B}=F \circ \Phi .
$$

We say in this case that $\Phi$ leads system (1) into pseudo-normal form ( $\Psi$ NF in shorter). Notice that (4) is not in general Poincaré-Dulac normal form, since the term $(D \Phi)^{-1} \hat{B}$ in the new system

$$
\dot{\zeta}=N(\zeta)+(D \Phi(\zeta))^{-1} \hat{B}(\zeta)
$$

must not exhibit any particular form in general.

Our first result is that, in this situation, the vector fields $N, \hat{B}$ and the transformation $\Phi$ leading the original system into $\Psi$ NF are convergent in a neighborhood of the equilibrium.

However, we prefer to state the main result in a bit more general framework. Namely, the very well known Lyapunov's Theorem ${ }^{12}$ ensures that if we have an $n$-degrees of freedom analytic Hamiltonian system, with the origin being an equilibrium and eigenvalues $\pm \lambda_{1}, \pm \lambda_{2}, \ldots, \pm \lambda_{n}$ satisfying that

- $\lambda_{1}$ is purely imaginary,

- none of the quocients $\lambda_{2} \div \lambda_{1}, \ldots \lambda_{n} \div \lambda_{1}$ is an integer,

then there exists a one-parameter family of periodic orbits accumulating to the origin. In other words, one can prove the existence of a transformation leading such system into (Birkhoff) normal form with respect to the variables associated to the imaginary eigenvalue.

In our context, we have the following result, which represents the simplest situation where Lyapunov's Theorem would apply, that is, when the 
spectrum of the differential of the field at the equilibrium consists on two pairs of eigenvalues.

Theorem 1 Let us consider a real system

$$
\left\{\begin{array}{l}
\dot{x}=\lambda x+\hat{f}_{1}(x, y, p, q) \\
\dot{y}=-\lambda y+\hat{g}_{1}(x, y, p, q) \\
\dot{p}=\alpha q+\hat{f}_{2}(x, y, p, q) \\
\dot{q}=-\alpha p+\hat{g}_{2}(x, y, p, q)
\end{array}\right.
$$

where $x, y, p, q \in \mathrm{R}, \lambda \cdot \alpha \neq 0$ reals and $\hat{f}_{j}, \hat{g}_{j}$, for $j=1,2$, analytic functions in all their variables which start with terms of order, at least, 2. Assume the origin to be a saddle-center equilibrium point of this system. Then, there exists an analytic in a neighborhood of the origin transformation $X=\Phi(\chi)$, being $X=(x, y, p, q)$ and $\chi=(\xi, \eta, \mu, \nu)$, and analytic vector fields $N, \hat{B}$

$$
N=\left(\begin{array}{r}
\xi A_{1}\left(\xi \eta, \mu^{2}+\nu^{2}\right) \\
-\eta A_{1}\left(\xi \eta, \mu^{2}+\nu^{2}\right) \\
\nu A_{2}\left(\xi \eta, \mu^{2}+\nu^{2}\right) \\
-\mu A_{2}\left(\xi \eta, \mu^{2}+\nu^{2}\right)
\end{array}\right), \quad \hat{B}=\left(\begin{array}{c}
\xi b_{1}\left(\xi \eta, \mu^{2}+\nu^{2}\right) \\
\eta b_{1}\left(\xi \eta, \mu^{2}+\nu^{2}\right) \\
\nu b_{2}\left(\xi \eta, \mu^{2}+\nu^{2}\right) \\
\mu b_{2}\left(\xi \eta, \mu^{2}+\nu^{2}\right)
\end{array}\right)
$$

leading system (6) into $\Psi \mathrm{NF}$, that is, verifying

$$
D \Phi N+\hat{B}=F \circ \Phi,
$$

where $F=\left(\lambda x+\hat{f}_{1},-\lambda y+\hat{g}_{1}, \alpha q+\hat{f}_{2},-\alpha p+\hat{g}_{2}\right)$.

In the Hamiltonian case, the remainder term $\hat{B}$ vanishes, so we reobtain, consequently, Lyapunov's result. Moreover, from partial views of system (6) it is not difficult to extend the convergence of the $\Psi \mathrm{NF}$ procedure to the following cases: a) the origin being a hyperbolic or an elliptic equilibrium of an autonomous system of the plane; b) in a neighborhood of a hyperbolic periodic orbit.

Apart from the class of Hamiltonian systems, it is possible to extract interesting consequences from Theorem 1 if we apply it onto the family of Reversible systems. We say that a system $\dot{X}=F(X)$ is $\mathcal{G}$-reversible, $\mathcal{G}$ being an involution $\left(\mathcal{G}^{2}=I d\right.$ and $\left.\mathcal{G} \neq I d\right)$, if it is invariant under $X \longmapsto \mathcal{G}(X)$ and a reversion in the sense of time's arrow $(t \mapsto-t)$ (see ${ }^{11,17,16}$ and references therein). It turns out that $F$ satisfies $\mathcal{G}^{*} F=-F$. Commonly $\mathcal{G}$ is called a reversing involution and is, in general, non linear. In this work we assume involutions $\mathcal{G}$ to be analytic. A set which is invariant under the action of $\mathcal{G}$ is called $\mathcal{G}$-symmetric.

Thus, in this Hamiltonian-Reversible context, we have 
Theorem 2 Let us consider an analytic system

$$
\dot{X}=F(X)
$$

and assume one of the following three situations holds,

(i) $X=(p, q) \in \mathrm{R}^{2}$ and the origin is a linear center equilibrium point.

(ii) $X=(x, y, \theta) \in \mathrm{R}^{2} \times \mathrm{T}$ and $\gamma=\{x=y=0\}$ is a (symmetric) hyperbolic periodic orbit.

(iii) $X=(x, y, p, q) \in \mathrm{R}^{4}$ and the origin is a saddle-center equilibrium point.

Then, in a neighborhood of the corresponding equilibrium, the following statements are equivalent

(i) System (8) is Hamiltonian.

(ii) System (8) is reversible.

(iii) The analytic vector field $\hat{B}$ provided by theorem 1 vanishes. (Notice that is means that the corresponding scalar functions $b_{2}, b_{1}$ or both, vanish, respectively).

\section{Acknowledgments}

Both authors are supported in part by the Spanish grant BFM2000-805 and the Catalan grant 2000SGR-27.

\section{References}

1. V.I. Arnold (Springer-Verlag, Berlin, 1982)

2. V.I. Arnold and Yu.S. Il'yashenko in Dynamical systems I, ed. D.V. Anosov and V.I. Arnold (Springer-Verlag, Berlin, 1988)

3. D. Bambusi, G. Cicogna, G. Gaeta and G. Marmo, J. Phys. A, 31, 5065, (1998).

4. G.R. Belitskiǐ, Funct. Anal. Appl., (20) 4, 253, (1986)

5. A.D. Bruno, Trans. Moscow Math. Soc., 25, 131, (1971)

6. A.D. Bruno (Springer-Verlag, Berlin, 1989)

7. G. Cicogna and G. Gaeta (Springer-Verlag, Berlin, 1999)

8. D. DeLatte, Ergod. Th. and Dynam. Sys., 15, 49, (1995)

9. A. Delshams, A. Guillamon and J.T. Lázaro, to appear in Qualit. Th. Dyn. Systems, (2001) 
10. A. Delshams and J.T. Lázaro, in preparation (2001)

11. R.L. Devaney, Trans. Am. Math. Soc., 218, 89, (1976)

12. A.M. Lyapunov, Ann. Fac. Sci. Toulouse, (2) 9, 203, (1907)

13. D. Montgomery and L. Zippin (Interscience, New York, 1955)

14. J. Moser, Comm. Pure and Appl. Math. , 9, 673, (1956)

15. J. Moser, Comm. Pure and Appl. Math. , 11, 257, (1958)

16. J.A.G. Roberts and G.R.W. Quispel, Physics Reports, 2-3 (216), 63, (1992)

17. M.B. Sevryuk (Springer-Verlag, Berlin, 1986)

18. S.P. Tokarev, Diff. Equations, 13, 613, (1977) 